\title{
Intracranial abscess complicating diverticulitis with CT scan mimicking primary glioma
}

\author{
A.R. Dixon ${ }^{1}$, J.T. Holmes ${ }^{1}$ and A. Waters ${ }^{2}$ \\ ${ }^{1}$ Peterborough District Hospital, Thorpe Road, Peterborough PE3 6DA and ${ }^{2}$ Addenbrooke's Hospital, Hills Road, \\ Cambridge CB2 2QQ, UK.
}

\begin{abstract}
Summary: The case of a 64 year old woman with diverticulitis complicated by a metastatic cerebral abscess is reported. Presentation was atypical and investigations were misleading; the computed tomographic scan was interpreted as showing a glioma. This case demonstrates that malignancy should never be assumed and the importance of making a histological diagnosis. Once the abscess was drained the patient made a full recovery with resolution of her neurological deficits.
\end{abstract}

\section{Introduction}

Although comprising only a small part of neurological practice with an incidence in the United Kingdom of approximately 4 per million per year, ${ }^{1}$ brain abscess remains an important and potentially dangerous condition. Early recognition plays the greatest part in determining outcome rather than any other factor. Diagnosis depends upon (i) the presence of a preexisting focus of infection, (ii) evidence of raised intracranial pressure and (iii) evidence of a focal neurological disturbance. ${ }^{2}$ The four most common causes are middle ear disease $(60 \%),{ }^{1,3}$ paranasal sinus infections $(10 \%){ }^{4}$ penetrating brain trauma and haematogenous metastatic infection. In the latter group these abscesses are frequently multiple and are usually seen complicating pulmonary infection, congenital heart disease, infective endocarditis and dental sepsis. ${ }^{1}$ In about $10 \%$ of cases no primary source of infection can be identified. ${ }^{1}$ We report a case complicating diverticulitis where presentation was atypical and investigations were misleading.

\section{Case report}

A 64 year old right-handed woman was admitted as an emergency with a 3-week history of explosive diarrhoea, persistent mucus discharge per rectum, anorexia, lethargy and with a provisional diagnosis of rectal neoplasm. The patient also remarked that she

Correspondence: A.R. Dixon, F.R.C.S., Department of Surgery, Glenfield General Hospital, Groby Road, Leicester LE3 9QP, UK.

Accepted: 12 January 1989 was becoming confused and forgetful. Examination found her apyrexial, forgetful but with no focal neurological signs. Rectal examination revealed a large, right-sided, extrarectal, non-tender mass with a stenosis of the rectum along with some blood and mucus on the glove.

Investigations revealed haemoglobin $10.1 \mathrm{~g} / \mathrm{dl}$ with a hypochromic microcytic film, white cell count $17 \times 10^{9} / 1$, ESR $20 \mathrm{~mm} / \mathrm{h}$ and normal chest X-ray. Biopsy of the rectal swelling revealed non-specific inflammation. Apart from fluctuating confusion she remained well with resolution of her bowel symptoms. A barium enema revealed a stricture of the rectum, sigmoid diverticulosis with contrast material lying outside the bowel (Figure 1). A diagnosis of rectal neoplasm or diverticulitis, and pararectal abscess with resultant fibrosis was made.

Twenty four hours later she become increasingly confused with a fluent dysphasia, right hemiparesis and a right upper motor neurone facial weakness. Computerized tomography (CT) (Figures 2 and 3) showed a ring enhancing mass in the left frontal lobe with surrounding oedema and midline shift. This was interpreted as either a metastasis or cerebral abscess. She was given dexamethasone and some improvement followed. A neurological opinion felt the CT was more in keeping with a primary glioma. Six days later she started vomiting, became drowsy and irritable, developing a dense right hemiparesis with an extensor plantar response. She remained apyrexial showing no features of papilloedema or meningeal irritation.

She was transferred to the regional neurosurgical unit and the CT was interpreted as showing a glioma. Four days later through a left frontal burr hole $45 \mathrm{ml}$

(C) The Fellowship of Postgraduate Medicine, 1989 


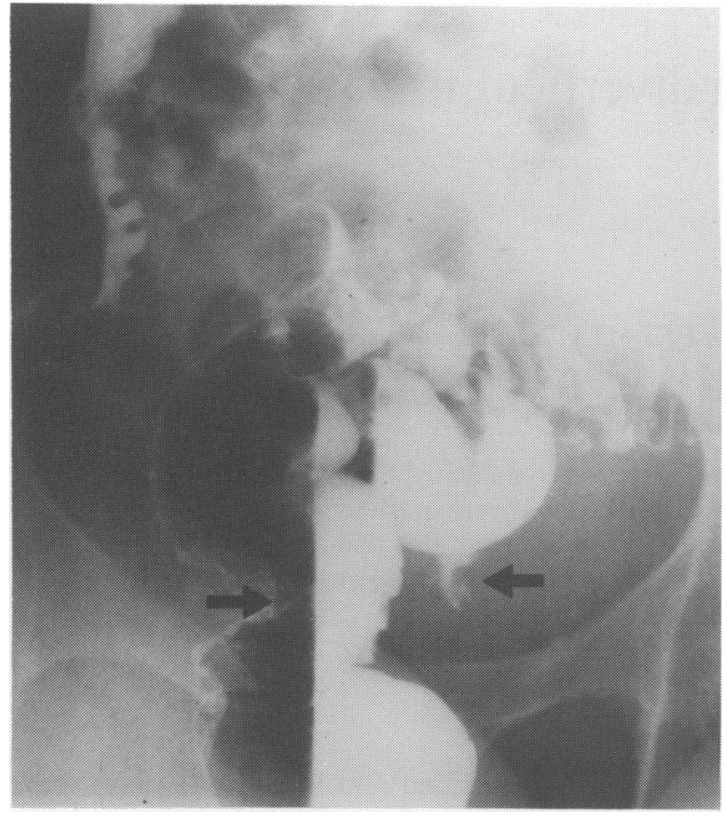

Figure 1 Barium enema showing sigmoid diverticulitis, extrarectal swelling and contrast lying outside the bowel (arrowed).

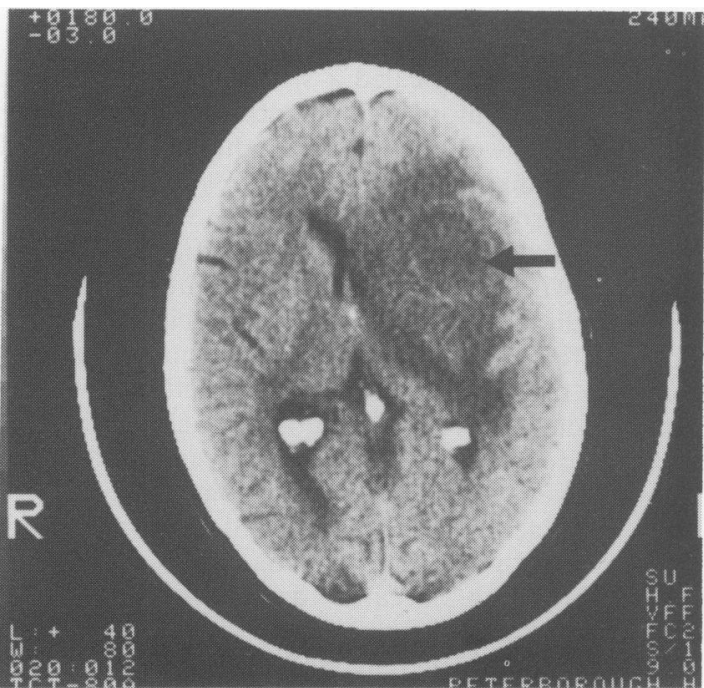

Figure 2 CT scan of head (without contrast) showing mass in left frontal lobe (arrowed) with surrounding oedema and midline shift.

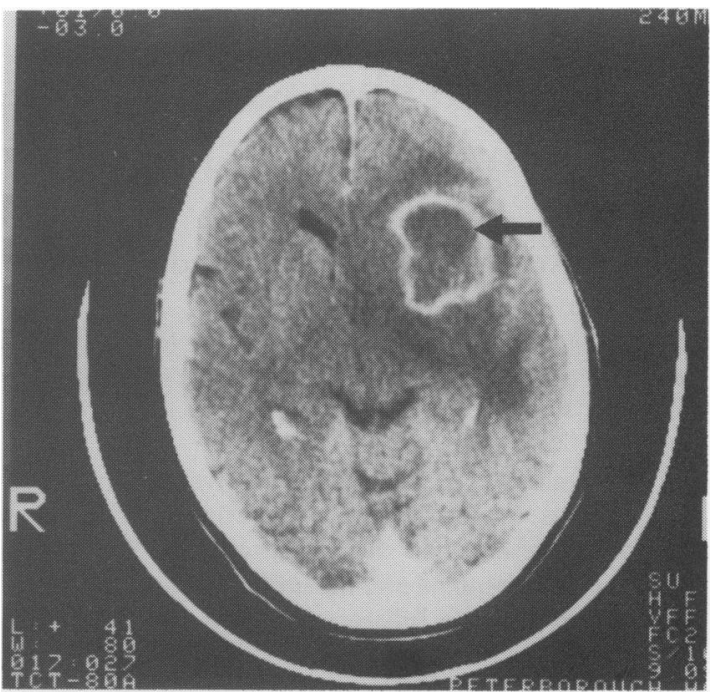

Figure 3 CT scan of head (following contrast injection) showing ring enhancing mass with surrounding oedema and midline shift.

of green pus was aspirated, and repeated 8 days later along with topical installation of gentamicin and vancomycin. A Lancefield group F streptococcus $(S$. milleri) was cultured, cefotaxime and metronidazole were given, and, by the 19 th postoperative day, the CT revealed complete resolution. The patient was alert and ambulant with resolution of the hemiparesis, dysphasia and rectal swelling. Indium-labelled leucocyte scans, sinus radiographs and an echcardiogram revealed no abnormality.

\section{Discussion}

Brain abscesses have been reported in two patients with abdominal sepsis (cholecystectomy and diverticulitis) suspected as being the aetiological factor. ${ }^{5}$ As there is no single specific presentation the diagnosis may not be obvious and a high level of clinical suspicion is required if the obvious consequence of a missed diagnosis is to be avoided. Post-mortem examination has provided the diagnosis in $29 \%$ of cases from one large series. ${ }^{5}$ Brain abscesses frequently present as expanding intracranial lesions with headache, epilepsy and focal neurological signs ${ }^{6,7}$ rather than as infectious processes, with fever frequently being absent..$^{6-8}$ However, despite the presence of a large abscess, symptoms and signs of raised intracranial pressure may be absent.

CT examination has become the most important 
means of investigation ${ }^{1,2}$ and along with swift referral, appropriate antibiotics and a high index of suspicion the prognosis has improved with survival rates of more than $90 \%$ now reported. ${ }^{9}$ However, as the above case illustrates, in the absence of an overt infectious process, differentiation between abscess and rapidly

\section{References}

1. Teddy, P.J. Intracranial abscess. In: Weatherall, D.J., Ledingham, J.G.G. \& Warrell, D.A. (eds) Oxford Textbook of Medicine, 2nd edition. Oxford University Press, Oxford, 1987, 21: pp. 152-155.

2. Garfield, J. Intracranial abscess. In: Rob, C. \& Smith, R. (eds) Operative Surgery: Neurosurgery, 3rd edition. Butterworth's, London, 1979, pp. 330-340.

3. Garfield, J. Management of supratentorial intracranial abscess; A review of 200 cases. Br Med J 1969, 2: 7-11.

4. Shaw, M.D.M. \& Russell, J.A. Cerebellar abscess - a review of 47 cases. J Neurol Neurosurg Psychiat 1975, 38: 429.

5. Brewer, N.S., MacCarty, C.S. \& Wellman, W.E. Brain abscess: a review of recent experience. Ann Int Med 1975, 82: $571-576$. growing malignant tumour may be impossible, CT interpretation can be difficult, the appearances of a cystic glioma being very similar. ${ }^{10}$ If a post-mortem diagnosis is to be avoided the presence of malignancy should never be assumed and histological confirmation is required.

6. Morgan, H., Wood, M.W. \& Murphey, F. Experience with 88 consecutive cases of brain abscess. $J$ Neurosurg 1973, 38: 698-704.

7. Samson, D.S. \& Clark, K. A current review of brain abscess. Am J Med 1973, 54: 201-210.

8. Gregory, D.H. \& Messner, R. \& Zinneman, H.H. Metastatic brain abscess: a retrospective appraisal of 29 patients. Arch Intern Med 1967, 119: 25-31.

9. Choudhury, A.R., Taylor, J.C. \& Whitaker, R. Primary excision of brain abscess. Br Med J 1977, 2: 1119-1121.

10. Ambrose, J., Gooding, M.R. \& Richardson, A.E. Sodium iothalamate as an aid to diagnosis of intracranial lesions by computerised transverse axial scanning. Lancet 1975, ii: 669. 